\title{
SOME WILD IMBEDDINGS IN CODIMENSION TWO
}

\author{
RALPH TINDELL ${ }^{1}$
}

\section{Statement of the Theorem.}

ThEOREM. For every $n \geqq 3$, the following exist in $E^{n}$ :

I. A wild $(n-2)$-sphere which is the union of two flat $(n-2)$-cells whose common boundary is a flat $(n-3)$-sphere.

II. A wild $(n-2)$-cell which is the union of two flat $(n-2)$-cells intersecting in a flat $(n-3)$-cell common to their boundaries.

III. A wild (n-2)-sphere which fails to be locally flat at precisely one point; moreover, it fails to be locally tame at that point.

IV. A wild (n-2)-cell which fails to be locally flat at precisely one point; moreover, the point is an interior point and the cell fails to be locally tame there.

The spheres in III were constructed by Cantrell and Edwards [2] as an example of a wild $(n-2)$-sphere which is locally flat at all save possibly one point; they asked whether or not the spheres were locally flat at that point, answered here in the negative. Examples I and II give examples of 2-complexes wildly imbedded in $E^{4}$ but such that each simplex is tamely imbedded; Edwards has shown [3] that for a 2-complex imbedded in $E^{n}$ for $n \geqq 6$, this cannot occur; for $n=5$, the question is open. Examples III and IV show that Theorems 3 and 6 of [3], proved for $n \geqq 6$, fail for $n=4$.

2. Definitions and lemmas. We regard $E^{k}, k \leqq n$, as the subset of $E^{n}$ defined by $x_{i}=0$ for all $i>k$, and define the positive half $k$-space $E_{+}^{\mathbf{k}}$ to be the subset of $E^{k}$ defined by $x_{k} \geqq 0$.

A topological $k$-manifold $M$ in $E^{n}$ is said to be locally flat at a point $p \in M$ if there is a neighborhood $U$ of $p$ (in $E^{n}$ ) and a homeomorphism $h$ carrying the pair $(U, U \cap M)$ onto the pair $\left(E^{n}, E^{k}\right)$ if $p \notin$ bdry $M$, or to $\left(E^{n}, E_{+}^{k}\right)$ if $p \in$ bdry $M . M$ is said to be locally tame at $p \in M$ if there is a neighborhood $U$ of $p$ (in $E^{n}$ ) and a homeomorphism $g$ of $U$ on to $E^{n}$ carrying $U \cap M$ onto a subpolyhedron of $E^{n} . M$ is said to be tame if there is a homeomorphism of $E^{n}$ onto itself carrying $M$ onto a subpolyhedron of $E^{n}$. A cell (sphere) in $E^{n}$ is said to be flat if there is

Presented to the Society, November 12, 1965; received by the editors May 12, 1965.

1 This research was partially supported by the National Science Foundation, Grant GP4006. The author wishes to express his gratitude for the help and encouragement of Professors O. G. Harrold, Jr., and J. J. Andrews. 
a homeomorphism of $E^{n}$ on to itself carrying the cell (sphere) onto a simplex (the boundary of a simplex).

The first lemma provides a precise way to prove that certain manifolds fail to be locally flat at a point (e.g., the cone over any nontrivial knot). It is a direct generalization of two theorems of Fox and Artin [5], the proof being essentially the same.

Lemma 1. If a k-manifold $M$ in $E^{n}, k \leqq n-2$, is locally flat at $p \in M$, then given any monotone sequence $V_{1} \supset V_{2} \supset \ldots$ of closed neighborhoods of $p\left(\right.$ in $\left.E^{n}\right)$ such that $\cap V_{i}=p$, there is an integer $N$ for which the image of $\pi_{1}\left(V_{N}-M\right)$ in $\pi_{1}\left(V_{1}-M\right)$, obtained by inclusion, is cyclic or trivial.

The next lemma occurs in [2], the proof being included here for completeness.

Leмma 2. If $P$ is a finite polyhedron in the $n$-sphere $S^{n}$, then $\pi_{1}\left(S^{n}-P\right)$ is finitely generated.

Proof. Let $N$ be a regular neighborhood of $P$ in $S^{n}$; then $S^{n}-\operatorname{Int} N$ is a deformation retract of $S^{n}-P$ and thus $\pi_{1}\left(S^{n}-P\right)$ is isomorphic to $\pi_{1}\left(S^{n}-\operatorname{Int} N\right)$. But $S^{n}-\operatorname{Int} N$ is a finite polyhedron and hence has finitely generated fundamental group.

Lemma 3. If a $k$-manifold $M$ in $E^{n}, k \leqq n-2$, is locally tame at $p \in M$, then for any $\epsilon>0$, there is a closed neighborhood $V$ of $p\left(\right.$ in $\left.E^{n}\right)$ of diameter less than $\epsilon$ such that $\pi_{1}(V-M)$ is finitely generated.

Proof. Since $M$ is locally tame at $p$, there is a neighborhood $U$ of $p$ and a homeomorphism $g: U \rightarrow E^{n}$ such that $g(U \cap M)$ is a polyhedron. If we could show that $g(p)$ had arbitrarily small neighborhoods $V^{\prime}$ for which $\pi_{1}\left(V^{\prime}-g(U \cap M)\right)$ is finitely generated, $g^{-1}\left(V^{\prime}\right)$ would then be the desired neighborhood of $p$. Thus we need only prove the lemma for $M$ a polyhedron, an assumption we now make.

Let $K$ be a complex with subcomplex $L$ such that $|K|=E^{n}$, $|L|=M$, and $p$ is a vertex of $K$. Since the closure of $M-|\operatorname{St}(p, L)|$ is a closed set missing $p$ there is a $\delta$ such that the $\delta$-neighborhood $S(p, \delta)$ of $p$ (in $\left.E^{n}\right)$ misses $\mathrm{Cl}(M-|\mathrm{St}(p, L)|)$; i.e., $S(p, \delta) \cap M$ $=S(p, \delta) \cap|\operatorname{St}(p, L)|$. Now let $K^{\prime}$ be a subdivision of $K$ of mesh less than $\min \{\delta / 2, \epsilon / 2\}$ and define $V$ to be $\left|\operatorname{St}\left(p, K^{\prime}\right)\right|$; then $V$ is a neighborhood of $p$ and $V \cap M=V \cap|\operatorname{St}(p, L)|$. From this and the definition of $V$ it is not difficult to see that $V \cap M(=V \cap|\operatorname{St}(p, L)|)$ is the join of (bdry $V) \cap M(=($ bdry $V) \cap|\operatorname{St}(p, L)|)$ with $p$. We may thus define a deformation retraction of $V-M$ onto (bdry $V)-M$ by projection from $p$; hence $\pi_{1}(V-M)$ is isomorphic to $\pi_{1}(($ bdry $V)-M)$. 
But bdry $V=$ bdry $\left|\operatorname{St}\left(p, K^{\prime}\right)\right|$ is a combinatorial $(n-1)$-sphere and (bdry $V) \cap M$ is a finite subpolyhedron so by Lemma $2, \pi_{1}$ ((bdry $V$ ) $-M$ ) is finitely generated. Finally, since $V$ is the star of a vertex in a complex of mesh less than $\epsilon / 2$, the diameter of $V$ is less than $\epsilon$.

A slight modification of the proof to Theorem 1 of [1] establishes the following lemma. Let $a$ and $b$ be the points in $E^{n}$ defined by $a_{i}=b_{i}=0$ for $i<n$, and $a_{n}=1, b_{n}=-1$.

Lemma 4. If $C$ is a compact set in $E^{n-1}$ and $T$ is the join of $C$ with $a \cup b$, then the injection $i_{*}: \pi_{1}\left(E^{n-1}-C\right) \rightarrow \pi_{1}\left(E^{n}-T\right)$ is an isomorphism onto ( $i_{*}$ induced by inclusion).

We now describe, for future reference, a sequence of closed neighborhoods of the vertex $a$, where $a, b, c$, and $T$ are as in Lemma 4 and it is assumed that $C$ contains the origin. The set $A_{i}$ is to be the $n$ cube with "bottom" face parallel to $E^{n-1}$, having the vertex $a$ at its center and edge length $2 \gamma_{i}$, where $\gamma_{i}=\operatorname{diam}\left(T \cap P_{i}\right), P_{i}$ being the horizontal $(n-1)$-plane defined by $x_{n}=i / i+1$. The following facts follow from the construction:

(i) $A_{1} \supset A_{2} \supset \cdots$,

(ii) $\cap A_{i}=p$,

(iii) $\pi_{1}\left(A_{1}-T\right)$ is isomorphic to $\Pi_{1}\left(E^{n}-C\right)$,

(iv) the injection $i_{*}: \pi_{1}\left(A_{j}-T\right) \rightarrow \pi_{1}\left(A_{1}-T\right)$ is an isomorphism onto.

Moreover, it is clear that the $A_{i}$ 's would still satisfy (i)-(iv) if $T$ were replaced with the cone over $C$ with vertex $a$.

\section{Proof of the Theorem.}

(3.1) The starting point of all of our examples is the Fox-Artin mildly wild arc (Example 1.4 of [4]) with its end-points joined by polygonal arcs to a point $q$ to form a 1 -sphere. $S$ is the union of the two tame arcs $D_{1}$ and $D_{2}$ running from $q$ to the bad point $p$ of the original arc. We note the following facts:

1. $\pi_{1}\left(E^{3}-S\right)$ is infinitely generated.

2. The taming homeomorphism for each $D_{i}$ may be chosen so that it is the identity outside some 3-cell.

3. $S$ fails to be locally tame at $p$.

Fox and Artin actually show (3) in the process of proving that their arc is wild and an examination of the proof will yield a presentation of $\pi_{1}\left(E^{3}-S\right)$ with generators $c_{i}, i=1,2, \cdots$, and relations $c_{i} c_{i+1} c_{i}$ $=c_{i+1} c_{i} c_{i+1}$; then, the map carrying $c_{i}$ to the cycle $(i i+1)$ defines a homomorphism of $\pi_{1}\left(E^{3}-S\right)$ onto an infinitely generated subgroup of the permutations of the positive integers. Thus $S$ satisfies (1) as 
claimed. Claim (2) follows from a result of Moise [6, Theorem 5].

(3.2) We establish Part I of the Theorem by proving the following

Proposition 1. For every $n \geqq 3$, there is an $(n-2)$-sphere $S$ in $E^{n}$ such that:

(i) $\pi_{1}\left(E^{n}-S\right)$ is infinitely generated.

(ii) $S=D_{1} \cup D_{2}$, where each $D_{i}$ is a flat $(n-2)$-cell with taming homeomorphism $\phi_{i}$ which is the identity outside some $n$-cell $F_{i}$.

(iii) $D_{1} \cap D_{2}=$ bdry $D_{1}=$ bdry $D_{2}$ is a flat polyhedral $(n-3)$-sphere.

Proof (By INDUCTION on $n$ ). The 1-sphere of (3.1) satisfies (i)-(iii), so the proposition is true for $n=3$.

Suppose now that $S$ is an $(n-3)$-sphere in $E^{n-1}$ satisfying conditions (i)-(iii). Let $T=\operatorname{Susp}(S)$, i.e., the join of $S$ with $a \cup b$ as in Lemma $4 ; T$ is an $(n-2)$-sphere and by Lemma $4, \pi_{1}\left(E^{n}-T\right)$ is isomorphic to $\pi_{1}\left(E^{n-1}-S\right)$ which is infinitely generated by hypothesis. Thus $T$ satisfies (i). Moreover, $T=\operatorname{Susp}\left(D_{1}\right) \cup \operatorname{Susp}\left(D_{2}\right)$ and $\operatorname{Susp}\left(D_{i}\right)$ is an $(n-2)$-cell. Now the taming homeomorphism $\phi_{i}: F_{i} \rightarrow F_{i}$ is the identity on the boundary of $F_{i}$ so the extension $\bar{\phi}_{i}: \operatorname{Susp}\left(F_{i}\right) \rightarrow \operatorname{Susp}\left(F_{i}\right)$ will be the identity on the boundary of the $n$-cell Susp $F_{i}$. We may thus extend $\bar{\phi}_{i}$ to all of $E^{n}$ by letting it be the identity off $\operatorname{Susp}\left(F_{i}\right)$ and $\bar{\phi}_{i}$ carries $\operatorname{Susp}\left(D_{i}\right)$ on to the suspension of an $(n-3)$-simplex which is of course a flat $(n-2)$-cell; thus $T$ satisfies (ii). Finally $\operatorname{Susp}\left(D_{1}\right) \cap \operatorname{Susp}\left(D_{2}\right)=\operatorname{Susp}\left(D_{1} \cap D_{2}\right)$ is a flat polyhedral $(n-4)$ sphere. Thus $T$ satisfies (iii) and the induction is complete.

Since condition (i) contradicts the conclusion of Lemma 2, $S$ cannot be tame and we have proved Part I of the Theorem.

(3.3) Let $S$ be an $(n-3)$-sphere satisfying (i)-(iii) as in Proposition 1 and let $D$ be $C(S)$, the cone over $S$ with vertex $a . D$ $=C\left(D_{1}\right) \cup C\left(D_{2}\right)$ and $C\left(D_{i}\right)$, by the arguments given for (ii) above, will be a flat $(n-2)$-cell; similarly $C\left(D_{1}\right) \cap C\left(D_{2}\right)$ will be a flat $(n-3)$-cell.

To see that $D$ is wild we show that it fails to be locally tame at the vertex $a$. Let $A_{i}$ be the closed neighborhoods of $a$ as defined after Lemma 4 , and let $\epsilon$ be small enough so that any neighborhood of diameter less than $\epsilon$ will be contained in $A_{1}$. Then any such neighborhood $V$ will contain some $A_{N}$ for $N$ large enough (recall that $A_{1} \supset A_{2} \supset \cdots$ and $\left.\cap A_{i}=a\right)$. The commutativity of the inclusion diagram

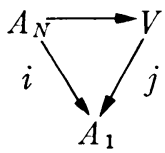

implies the commutativity of the induced injection diagram 


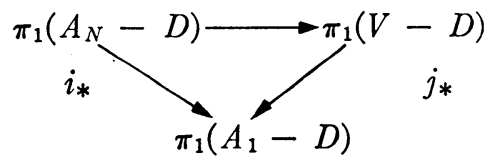

and since $i_{*}$ is onto, $j_{*}$ must be onto. But $\pi_{1}\left(A_{1}-D\right) \approx \pi_{1}\left(E^{n-1}-S\right)$ is infinitely generated. We have shown then that for every neighborhood $V$ of $p$ of diameter less than $\epsilon, \pi_{1}(V-D)$ is infinitely generated; thus, by Lemma $3, D$ cannot be locally tame at $a$ and hence cannot be tame. This established Part II of the Theorem.

(3.4) Proposition 2. For every $n \geqq 3$, there is an $(n-2)$-sphere $S$ in $E^{n}$ such that:

(i) $\pi_{1}\left(E^{n}-S\right)$ is infinitely generated.

(ii) $S$ is locally flat except at a single point $p$.

(iii) $S$ fails to be locally tame at $p$.

Proof (Induction on $n$ ). The 1 -sphere given in (3.1) satisfies the conditions of the proposition which is thus true for $n=3$.

Suppose now that $S^{\prime}$ is an $(n-3)$-sphere in $E^{n-1}$ satisfying conditions (i)-(iii) above. Let $T=\operatorname{Susp}\left(S^{\prime}\right)$ and $L=\operatorname{Susp}\left(p^{\prime}\right)$. By Lemma 4, $\pi_{1}\left(E^{n}-T\right)$ is isomorphic to $\pi_{1}\left(E^{n-1}-S^{\prime}\right)$ and hence is infinitely generated. Clearly $T$ will be locally flat except possibly at the points of the $\operatorname{arc} L$. Since $L$ is cellular in both $E^{n}$ and $T$, shrinking $L$ to a point will result in an $(n-2)$-sphere $S=T / L$ in a space $E^{n} / L$ which is homeomorphic to $E^{n}$; let $f$ be the shrinking map of $E^{n}$ onto $E^{n} / L$. $T$ is locally flat off $L$ so $S$ will be locally flat except possibly at the point $p=f(L)$, recalling that $f$ is a homeomorphism off $L$. And hence $\pi_{1}\left(E^{n}-S\right)$ will be infinitely generated because $f$ will induce an isomorphism carrying it onto $\pi_{1}\left(E^{n}-T\right)$; we have shown then that $S$ satisfies (i).

Now consider the following sequence of closed neighborhoods of the arc $L: V_{1}=A_{i} \cup B_{i} \cup F_{i}$, where $A_{i}$ is the neighborhood of the vertex $a$ as described after Lemma $4, B_{i}$ is the closed $(1 / i+1)$-neighborhood of the vertex $b, F_{1}$ is the suspension of an $(n-1)$-cell containing all of $S^{\prime}$ in its interior, and $F_{i}$ is the suspension of the closed $(1 / i)$ neighborhood of the bad point $p^{\prime}$ of $S^{\prime}$ (in $E^{n-1}$ ), $i \neq 1$. Clearly each $V_{i}$ is a closed neighborhood of $L, \cap V_{i}=L$, and $V_{1} \supset V_{2} \supset \ldots$. Moreover the commutativity of the diagram

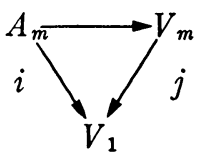

implies the commutativity of the injection diagram 


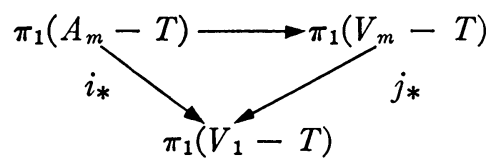

and since $\pi_{1}\left(V_{1}-T\right) \approx \pi_{1}\left(E^{n}-T\right)$ and $i_{*}$ is onto, $j_{*}$ maps $\pi_{1}\left(V_{m}-T\right)$ onto the infinitely generated group $\pi_{1}\left(V_{1}-T\right)$. Defining $V_{i}^{\prime}=f\left(V_{i}\right)$ we obtain a sequence $V_{1}^{\prime} \supset V_{2}^{\prime} \supset \ldots$ of closed neighborhoods of $p^{\prime}$ such that $\cap V_{i}^{\prime}=p^{\prime}$; finally, the injection $i_{*}: \pi_{1}\left(V_{m}^{\prime}-S\right) \rightarrow \pi_{1}\left(V_{1}^{\prime}-S\right)$ is onto and $\pi_{1}\left(V_{1}^{\prime}-S\right)$ is infinitely generated (remembering that $f$ is a homeomorphism off $L$ ). Since this violates the conclusion of Lemma 1, $S$ cannot be locally flat at $p$. Using this sequence of neighborhoods, the arguments used in (3.3) show that $S$ fails to be locally tame at $p$ and the induction is complete. This establishes Part III of the Theorem.

(3.5) To obtain an $(n-2)$-cell as described in Part IV of the Theorem, we consider the sphere $T$ constructed in (3.4) and remove a nice cell that misses $L$ before we shrink $L$ to a point. All the arguments above are still valid and the result will be an $(n-2)$-cell $D$ which is locally flat at every point except $p, p$ is an interior point of $D$, and $D$ fails to be locally tame at $p$. We have thus completed the proof of the Theorem.

CONJECTURE. The cells of Part IV are mildly wild; i.e., there is an interior point $P$ of the $(n-2)$-cell such that any $(n-2)$-cell subset having $P$ on its boundary is tame.

The author has since proved the conjecture for the case $n=4$, solving a problem proposed by R. H. Fox [5, Problem 21].

Added in proof. We have recently learned that Sossinskiu has independently obtained similar examples.

\section{REFERENCES}

1. J. J. Andrews and M. L. Curtis, Knotted 2-spheres in the 4-sphere, Ann. of Math. 70 (1959), 565-571.

2. J. C. Cantrell and C. H. Edwards, Jr., Almost locally flat imbeddings of manifolds, Michigan Math. J 12 (1965), 217-223.

3. C. H. Edwards, Jr., Taming 2-complexes in high dimensional manifolds, Duke Math J. 32 (1965), 479-494.

4. R. H. Fox and E. Artin, Some wild cells and spheres in three space, Ann. of Math. 49 (1948), 979-990.

5. R. H. Fox, Some problems in knot theory, Topology of 3-manifolds, pp. 168-176, ed. by M. K. Fort, Jr., Prentice-Hall, Englewood Cliffs, N. J., 1962. 114.

6. E. E. Moise, Affine structures in 3-manifolds (V), Ann. of Math. 56 (1952), 96- 